\title{
New Galactic Wolf-Rayet stars, and candidates (Research Note)
}

\section{An annex to The VIIth Catalogue of Galactic Wolf-Rayet Stars}

\author{
K. A. van der Hucht ${ }^{1,2}$ \\ 1 SRON Netherlands Institute for Space Research, Sorbonnelaan 2, 3584CA Utrecht, The Netherlands \\ e-mail: k.a.van.der.hucht@sron.nl \\ 2 Astronomical Institute Anton Pannekoek, University of Amsterdam, Kruislaan 403, 1097 SJ Amsterdam, The Netherlands \\ Received 13 June 2006 / Accepted 13 July 2006
}

\section{ABSTRACT}

This paper gathers, from the literature and private communication, 72 new Galactic Population I Wolf-Rayet stars and 17 candidate WCLd stars, recognized and/or discovered after the publication of The VIIth Catalogue of Galactic Wolf-Rayet Stars. This brings the total number of known Galactic Wolf-Rayet stars to 298, of which $24(8 \%)$ are in open cluster Westerlund 1, and 60 (20\%) are in open clusters near the Galactic Center.

Key words. stars: Wolf-Rayet

\section{Introduction}

Wolf-Rayet (WR) stars represent the final phase in the evolution of massive stars (i.e., $M_{i} \gtrsim 20 M_{\odot}$ ), before becoming a supernova and/or stellar remnant. They are the chemically evolved descendants of OB stars (e.g., Meynet \& Maeder 2005) and contribute to chemical and kinetic enrichment of their environment through their dense stellar winds and Lyman continuum photons. Some of them could be the possible progenitors of core-collapse supernovae and $\gamma$-ray bursts, especially in a low metallicity environment (e.g., Hirschi et al. 2005; Petrovic et al. 2005; Yoon \& Langer 2005; Langer \& Norman 2006; Woosley \& Heger 2006; Fruchter et al. 2006). Where $35 \%$ of the Galactic WR stars have wind blown bubbles, visible as ring nebulae (Marston 1997), they provide the ideal environment for a $\gamma$-ray burst afterglow (e.g., Chevalier 2005; Dwardakas 2005; Zou et al. 2005; Eldridge et al. 2006; Eldridge \& Vink 2006; Hammer et al. 2006). For all practical purposes, it is important to know as many WR stars as possible. Assembling a complete catalogue of WR stars, their spectral types (and hence chemical make-up) and relative numbers is important in order to understand their impact on the Galactic environment as well as to investigate their suitability as precursors to very energetic processes in extragalactic systems.

Since the publication of The VIIth Catalogue of Galactic Wolf-Rayet Stars (van der Hucht 2001, henceforth 7Cat), numerous new Galactic Population I Wolf-Rayet stars have been discovered, notably near the Galactic Center (in the infrared) and in open clusters (e.g., in Westerlund 1, optically), but also as individual field stars, thanks to the advancements in sensitivity and spatial resolution. In order to list these new WR stars properly in the 7Cat numbering system, and because of the crowding and the occasional resolution of apparently single objects into multiple objects, it became necessary to have the RA/Dec(J2000) coordinates of the 26 7Cat WR stars near the Galactic Center re-determined with higher accuracy. For example, with improving spatial resolution and sensitivity, it appears that what Krabbe et al. (1995) saw as the single object GC IRS 13E (=WR 101f in 7Cat, WN9-10), has been resolved by Maillard et al. (2004) into a cluster containing 7 stars, including two WR stars (GC IRS13 E2 and GC IRS13 E4) and three candidate WCLd stars (GC IRS13 E3A, GC IRS13 E3B, and GC IRS13 E5). A critical analysis of IRS 13E has been presented recently by Paumard et al. (2006).

This paper, rather than providing a completely revised WR catalogue, presents as an annex to the 7Cat a list of new WR stars and candidate WR stars discovered in recent years, together with updated coordinates for some objects.

All of the new discoveries quoted here require confirmation by additional multi-frequency high-spectral resolution and highangular resolution observations, which may throw new light on earlier results, e.g., Tanner et al. (2006). For example, GC IRS8, one of five GC stars suggested by Tanner et al. (2005) to be WR stars, turned out to be an O5-O6 giant or supergiant when observed by Geballe et al. (2006).

\section{New data}

The new Galactic WR stars listed in this annex have been discovered by the following authors:

- Some 15 possible WR stars in the Arches cluster had been recognized (in the infrared) before 2001 by Nagata et al. (1995) and Cotera et al. (1996), as noted by Blum et al. (2001), Lang et al. (2001), and Figer et al. (2002). Those objects are now included in this annex.

- Bartaya et al. (1994) discovered (in the optical) one new WN4 star (WR 159) in the OB association Cas OB 4, which has been re-discovered by Negueruela (2003).

- Figer et al. (1996) discovered (in the infrared) two WN9/Ofpe and five possible WCLd stars in the Quintuplet cluster. Tuthill et al. (2006) showed that at least two of those 
WCLd stars, Q2 and Q3, have infrared pinwheels, indicative of dust formation originating in the colliding winds of long period WCL+OB binaries.

- Clark \& Negueruela (2002), Negueruela \& Clark (2003), Clark et al. (2005), Negueruela \& Clark (2005), Negueruela (priv. comm.), and Crowther et al. (2006), discovered (in the optical) 24 new WR stars in the open cluster Westerlund 1, extremely rich in O stars, WR stars and LBVs. Groh et al. (2006) independently discovered (in the optical) three $\mathrm{W} \mathrm{d} 1$ WR stars discovered also by Crowther et al. (2006).

- Pasquali et al. (2002) discovered (in the infrared) one new WC8 star (WR 142a), in Cygnus.

- Homeier et al. (2003) discovered (in the infrared) three WC8-9 stars and one WN10 star, in the inner Galaxy.

- Drew et al. (2004) discovered (in the optical) one new WO3 star (WR 93b), located most likely in the Scutum-Crux arm of the inner Milky Way, from follow-up observations of candidate emission-line stars in the AAO/UKST Southern Galactic Plane $\mathrm{H} \alpha$ Survey (Parker et al. 2005).

- Cohen et al. (2005) discovered (in the optical and infrared) one new WN7 star (WR 75ab), from follow-up observations of candidate emission-line stars in the AAO/UKST Southern Galactic Plane H $\alpha$ Survey (Parker et al. 2005).

- Hopewell et al. (2005) discovered (in the optical) five new WC9 stars in a programme of follow-up optical spectroscopy of candidate emission-line stars in the AAO/UKST Southern Galactic Plane $\mathrm{H} \alpha$ Survey (Parker et al. 2005).

- Paumard et al. (2001, 2006), Eckart et al. (2004), Horrobin et al. (2004), Maillard et al. (2004), Moultaka et al. (2005), and Tanner et al. $(2002,2005)$ together discovered (in the infrared) 14 new WR stars and 14 candidate WCLd stars in the Galactic Center cluster.

- Eikenberry et al. (2001, 2004) discovered (in the infrared) one new WC9 star (WR 111b) in the cluster apparently near the soft $\gamma$-ray repeater SGR 1806-20.

- Figer et al. (2005) discovered (in the optical and infrared) three new WR stars in the cluster around the soft $\gamma$-ray repeater SGR 1806-20, two of which (WR 111a and WR 111c) had been discovered independently (in the infrared) by LaVine and Eikenberry (2004, private communication).

\section{The census of Population I WR stars and candidate WR stars in the Galaxy}

The new Galactic WR stars and candidates are listed in Table 1, together with those WR stars from the 7Cat for which the coordinates have been re-determined. Table 1 lists:

- Galactic WR running number in the 7Cat system;

- WR discovery designation, acknowledging the authors of the discovery paper;

- additional designation(s) from the literature;

- discovery spectral type;

- revised spectral type;

- magnitude $(V$, or $R$, or $K)$;

- RA/Dec(J2000) coordinates;

- discovery reference.

There are 72 new Galactic WR stars listed in this annex, plus 17 candidate WR stars, some possibly of the WCLd type. Of the 72 new Galactic WR stars we find: 45 WN stars, 26 WC stars, and one WO star.

Of the 72 new Galactic WR stars, in most cases the number of observations is still too small to establish which are binaries.
We would expect a binary frequency of $\sim 40 \%$ (van der Hucht 2001, Table 20). Only a few new WR stars have shown some indication of binarity (see Table 1).

There are now 60 known WR stars in the open clusters near the Galactic Center, i.e., the Galactic Center cluster (29, plus 13 candidate WR stars), the Arches Cluster (17, all WN) and the Quintuplet cluster (14, plus 3 candidate WR stars), plus 16 candidate WR stars, mostly candidate WCLd.

Together with the 226 WR stars in the 7Cat, this annex brings the total number of presently known Galactic Population I WR stars to 298 , excluding the 17 candidate WR stars. The spectral subtype distribution is: $171 \mathrm{WN}$ stars, $10 \mathrm{WN} / \mathrm{WC}$ stars, 113 WC stars, and 4 WO stars.

The 7Cat has 53 of its 226 WR stars in open clusters and OB associations, i.e. $23 \%$. Together with this annex we count 137 out of the 298 known Galactic WR stars in open clusters and OB associations, i.e. $46 \%$, of which $8 \%$ are in open cluster Westerlund 1 and $20 \%$ are in open clusters near the Galactic Center.

\section{Notes on individual stars}

\section{Westerlund 1:}

77b $=$ NC-N: X-ray detection by Chandra (Skinner et al. 2006).

$\mathbf{7 7 g}=$ NC-K: X-ray detection by Chandra (Skinner et al. 2006). 77j = NC-G: X-ray detection by Chandra (Skinner et al. 2006). 77k = NC-L = Wd1-44: X-ray detection by Chandra (Skinner et al. 2006).

77n $=\mathrm{NC}-\mathrm{F}=\mathrm{Wd1-239:}$ X-ray detection by Chandra (Skinner et al. 2006).

77o = NC-B: X-ray detection by Chandra (Skinner et al. 2006). Relatively high $L_{\mathrm{x}}$, possibly colliding wind binary.

$\mathbf{7 7} \mathbf{p}=\mathrm{NC}-\mathrm{E}=\mathrm{Wd1-241:}$ X-ray detection by Chandra (Skinner et al. 2006).

$\mathbf{7 7 q}=\mathrm{NC}-\mathrm{R}=\mathrm{WD} 1-14 \mathrm{c}: \mathrm{X}-$ ray detection by Chandra (Skinner et al. 2006).

77r = NC-D: X-ray detection by Chandra (Skinner et al. 2006). 77sa $=$ NC-W = GDTB 3: X-ray detection by Chandra (Skinner et al. 2006).

77sb = NC-O: X-ray detection by Chandra (Skinner et al. 2006). 77sc = NC-A: X-ray detection by Chandra (Skinner et al. 2006). Relatively high $L_{\mathrm{x}}$, possibly colliding-wind binary.

\section{Galactic Center cluster:}

WR 101b = AF-NW: tentative association with X-ray source CXOGC/J174539.4-2900310 (Baganoff et al. 2003).

WR 101db = GC IRS 34W: irregular variable $(\Delta K=1.5 \mathrm{mag})$, possibly indicative for LBV phase (Trippe et al. 2006).

WR 101fa = GC IRS 3: an ESO VLTI-MIDI observation by Pott et al. (2005) shows a $N$-band $(8-12 \mu \mathrm{m})$ size of $\leq 40$ mas, i.e., $\leq 300 \mathrm{AU}$, compatible with the typical dust envelope size of WCd stars (Williams et al. 1987). However, Pott et al. argue that the WC5-6 spectrum may be associated with a faint star $\sim 120$ mas east of IRS 3. See also Viehmann et al. (2006).

WR 101k = GC IRS 16SW: periodic IR variable, $K$-band light curve, $P=9.725 \mathrm{~d}, M \simeq 100 M_{\odot}$ (Ott et al. 1999; De Poy et al. 2004).

WR 101nd = GC IRS 16NE: RV variable, may be SB (Tanner et al. 2006). 
Table 1. New Galactic Wolf-Rayet stars. Data quoted from 7Cat are listed in italics, revised and new data are listed in roman font.

\begin{tabular}{|c|c|c|c|c|c|c|c|c|c|c|c|}
\hline WR & $\begin{array}{l}\text { WR } \\
\text { discovery } \\
\text { designation }\end{array}$ & Other designation(s) & $\begin{array}{l}\text { Discovery } \\
\text { spectral } \\
\text { type } \\
\end{array}$ & $\begin{array}{l}\text { Revised } \\
\text { spectral } \\
\text { type }\end{array}$ & ref. & $\begin{array}{c}m \\
(\mathrm{mag})\end{array}$ & ref. & $\mathrm{RA}(\mathrm{J} 2000)$ & $\operatorname{Dec}(J 2000)$ & ref. & $\begin{array}{l}\text { WR } \\
\text { discovery } \\
\text { ref. }\end{array}$ \\
\hline $75 \mathrm{aa}$ & HBD 1 & SHS J162620.2-455946 & WC9d & & & $I=14.18$ & HB05 & 162620.2 & -455946 & HB05 & HB05 \\
\hline $75 \mathrm{ab}$ & CPG 1 & & WN7h & & & $K_{\mathrm{s}}=8.91$ & CP05 & 163348.74 & -492843.5 & $\mathrm{CP} 05$ & $\mathrm{CP} 05$ \\
\hline $75 \mathrm{c}$ & HBD 2 & SHS J163403.6-434025 & WC9 & & & $I=13.12$ & HB05 & 163403.6 & -434025 & HB05 & HB05 \\
\hline $75 d$ & HBD 3 & SHS J163417.5-460852 & WC9 & & & $I=12.30$ & HB05 & 163417.5 & -460852 & HB05 & HB05 \\
\hline
\end{tabular}

\section{open cluster Westerlund 1}

\begin{tabular}{|c|c|c|c|c|c|c|c|c|c|c|c|}
\hline $77 \mathrm{aa}$ & HBD 4, NC-T & SHS J164646.3-454758 & WC9d & & & $J=10.04$ & 2MASS & 164646.3 & -454758 & HB05 & HB05 \\
\hline $77 \mathrm{a}$ & $\mathrm{NC}-\mathrm{Q}$ & & WN6-7 & WN6 & CH06 & $J=11.72$ & CH06 & 164655.55 & -455135.0 & $\mathrm{CH} 06$ & $\mathrm{NC} 05$ \\
\hline $77 \mathrm{~b}$ & $\mathrm{NC}-\mathrm{N}$ & & WC8 & WC9d & CH06 & $J=9.69$ & 2MASS & 164659.9 & -455526 & 2MASS & $\mathrm{NC} 05$ \\
\hline $77 \mathrm{c}^{1}$ & NC-I & & WN6-8 & WN8 & CH06 & $J=10.89$ & CH06 & 164700.88 & -455120.8 & CH06 & $\mathrm{CN} 02, \mathrm{NCO} 3$ \\
\hline $77 d$ & NC-P & Wd1-57c & WN8 & WN7 & $\mathrm{CH} 06$ & $J=11.06$ & $\mathrm{CH} 06$ & 164701.59 & -455145.5 & $\mathrm{CH} 06$ & $\mathrm{NC} 05$ \\
\hline $77 \mathrm{e}^{2}$ & NC-J & & WNL & WN5 & $\mathrm{CH} 06$ & $J=11.7:$ & $\mathrm{CH} 06$ & 164702.47 & -455100.1 & CH06 & $\mathrm{CN} 02, \mathrm{NCO} 3$ \\
\hline $77 f$ & NC-S & Wd1-5 & WNVL & WN10-11: & $\mathrm{CH} 06$ & $J=9.81$ & CH06 & 164702.98 & -455020.0 & CH06 & $\mathrm{NC} 05$ \\
\hline $77 g^{3}$ & $\mathrm{NC}-\mathrm{K}$ & & WC & WC8 & CH06 & $J=11.81$ & $\mathrm{CH} 06$ & 164703.25 & -455043.8 & CH06 & $\mathrm{NCO3}$ \\
\hline $77 \mathrm{~h}$ & $\mathrm{NC}-\mathrm{V}$ & & WN8 & & & $J=10.47$ & $\mathrm{CH} 06$ & 164703.81 & -455038.8 & CH06 & $\mathrm{Ne} 05$ \\
\hline $77 \mathrm{i}$ & NC-M & Wd1-66 & WC9 & WC9d & CH06 & $J=10.13$ & $\mathrm{CH} 06$ & 164703.96 & -455137.8 & CH06 & $\mathrm{NC} 05$ \\
\hline $77 j^{4}$ & NC-G & & WN6-8 & WN7 & $\mathrm{CH} 06$ & $J=11.35$ & $\mathrm{CH} 06$ & 164704.01 & -455125.2 & $\mathrm{CH} 06$ & $\mathrm{CN} 02, \mathrm{NC} 03$ \\
\hline $77 \mathrm{k}$ & NC-L & Wd1-44 & WN9 & WN9: & CH06 & $J=9.08$ & CH06 & 164704.19 & -455107.4 & CH06 & $\mathrm{NC} 05$ \\
\hline $771^{5}$ & $\mathrm{NC}-\mathrm{H}$ & & WC9 & WC9d & CH06 & $J=10.3:$ & $\mathrm{CH} 06$ & 164704.22 & -455120.2 & CH06 & $\mathrm{CN} 02, \mathrm{NCO} 3$ \\
\hline $77 \mathrm{~m}^{6}$ & $\mathrm{NC}-\mathrm{C}$ & & WC8 & WC9d & $\mathrm{NC} 05$ & $J=11.26$ & $\mathrm{CH} 06$ & 164704.40 & -455103.8 & CH06 & $\mathrm{CN} 02, \mathrm{NCO} 3$ \\
\hline $77 n^{7}$ & $\mathrm{NC}-\mathrm{F}$ & Wd1-239 & WC9 & WC9d & $\mathrm{CH} 06$ & $J=9.85$ & $\mathrm{CH} 06$ & 164705.22 & -455225.0 & $\mathrm{CH} 06$ & $\mathrm{CN} 02, \mathrm{NC} 03$ \\
\hline $770^{8}$ & NC-B & & WNL & WN7 & CH06 & $J=10.91$ & $\mathrm{CH} 06$ & 164705.36 & -455105.0 & CH06 & $\mathrm{CN} 02, \mathrm{NCO} 3$ \\
\hline $77 p^{9}$ & $\mathrm{NC}-\mathrm{E}$ & Wd1-241 & WC9 & & & $J=10.12$ & $\mathrm{CH} 06$ & 164706.05 & -455208.2 & CH06 & $\mathrm{CN} 02, \mathrm{NCO} 3$ \\
\hline $77 q$ & NC-R & Wd1-14c & WN6-7 & WN5 & $\mathrm{CH} 06$ & $J=11.92$ & $\mathrm{CH} 06$ & 164706.07 & -455022.6 & $\mathrm{CH} 06$ & $\mathrm{NC} 05$ \\
\hline $77 \mathrm{r}^{10}$ & NC-D & & WN6-8 & WN7 & CH06 & $J=11.63$ & $\mathrm{CH} 06$ & 164706.24 & -455126.5 & CH06 & $\mathrm{CN} 02, \mathrm{NCO} 3$ \\
\hline $77 \mathrm{~s} \quad\{$ & $\begin{array}{l}\text { NC-U } \\
\text { GDTB } 1\end{array}$ & & $\begin{array}{l}\text { WN4 } \\
\text { WN5-7 }\end{array}$ & WN6 & CH06 & $\begin{array}{l}J=10.76 \\
K=9.19\end{array}$ & $\begin{array}{l}\text { CH06 } \\
\text { GD06 }\end{array}$ & $\begin{array}{l}164706.55 \\
164706.6\end{array}$ & $\begin{array}{l}-455039.0 \\
-455038.6\end{array}$ & $\begin{array}{l}\text { CH06 } \\
\text { GD06 }\end{array}$ & $\begin{array}{l}\mathrm{Ne} 05 \\
\text { GD06 }\end{array}$ \\
\hline $77 \mathrm{sa} \quad\{$ & $\begin{array}{l}\text { NC-W } \\
\text { GDTB } 3\end{array}$ & & $\begin{array}{l}\text { WN5-6: } \\
\text { WN7 }\end{array}$ & WN6 & CH06 & $\begin{array}{l}J=12.11 \\
K=9.70\end{array}$ & $\begin{array}{l}\text { CH06 } \\
\text { GD06 }\end{array}$ & $\begin{array}{l}164707.58 \\
164707.6\end{array}$ & $\begin{array}{l}-454922.2 \\
-454921.7\end{array}$ & $\begin{array}{l}\text { CH06 } \\
\text { GD06 }\end{array}$ & $\begin{array}{l}\text { CH06 } \\
\text { GD06 }\end{array}$ \\
\hline $77 \mathrm{sb}$ & $\mathrm{NC}-\mathrm{O}$ & & WN6 & & & $J=11.00$ & $\mathrm{CH} 06$ & 164707.66 & -455235.9 & CH06 & $\mathrm{NC} 05$ \\
\hline $77 \mathrm{sc}^{11}$ & NC-A & Wd1-72 & WN4-5 & WN7 & CH06 & $J=10.34$ & CH06 & 164708.32 & -455045.5 & CH06 & $\mathrm{CN} 02, \mathrm{NCO} 3$ \\
\hline $77 \mathrm{sd} \quad\{$ & $\begin{array}{l}\text { NC-X } \\
\text { GDTB } 2 \\
\end{array}$ & & $\begin{array}{l}\text { WN4-5: } \\
\text { WN4-5 }\end{array}$ & WN5 & CH06 & $\begin{array}{l}J=12.36 \\
K=9.99 \\
\end{array}$ & $\begin{array}{l}\text { 2MASS } \\
\text { GD06 }\end{array}$ & $\begin{array}{l}164714.1 \\
164714.2 \\
\end{array}$ & $\begin{array}{lll}-4548 & 32 \\
-45 & 48 & 31.4 \\
\end{array}$ & $\begin{array}{c}\text { 2MASS } \\
\text { GD06 }\end{array}$ & $\begin{array}{l}\text { CH06 } \\
\text { GD06 } \\
\end{array}$ \\
\hline $77 \mathrm{t}$ & HBD 5 & SHS J165057.6-434028 & WC9d & & & $I=13.00$ & HB05 & 165057.6 & -434028 & HB05 & HB05 \\
\hline $93 b$ & DBU 1 & & WO3 & & & $K=10.17$ & DB04 & 173203.30 & -350432.5 & DB04 & DB04 \\
\hline
\end{tabular}

\section{Arches cluster:}

WR 102aa = NWS 1 = AR6: non-thermal radio source $($ Lang et al. 2001). X-ray detection (Wang et al. 2006). Maybe WN8+OB colliding wind binary.

WR 102ad $=$ NWS $4=$ AR3: moderately variable $(29 \%)$ radio source, possibly indicative of a colliding wind binary (Lang et al. 2005).

WR 102ae = NWS 5: source A2 in X-ray detection by Law \& Yusef-Zadeh (2004) and Wang et al. (2006).

WR 102ah $=$ NWS $8=$ AR 1 : source A1S in X-ray detection by Law \& Yusef-Zadeh (2004) and Wang et al. (2006). Non-thermal and moderately variable (12\%) radio source, possibly indicative of a colliding wind binary (Lang et al. 2005).

WR 102ai = NWS 9 = AR8: moderately variable $(25 \%)$ radio source, possibly indicative of a colliding wind binary (Lang et al. 2005).

WR 102aj = NWS $10=$ AR4: source A1N in X-ray detection by Law \& Yusef-Zadeh (2004) and Wang et al. (2006). Moderately variable $(30 \%)$ radio source, possibly indicative of a colliding wind binary (Lang et al. 2005).

WR 102b = Sgr A-A: X-ray detection (Muno et al. 2006).

\section{Quintuplet cluster:}

WR 102dc = Q2 = GCS3-2 = qF231 = QR7: variable at $K$ (Glass et al. 1999, 2001), indicative of WCLd+OB colliding wind binary. Detection in X-rays (Law \& Yusef-Zadeh 2004; Wang et al. (2006). IR pinwheel discovered (Tuthill et al. 2006), proving a WCLd+OB colliding wind binary.

WR 102ha $=\mathrm{Q} 3=$ GCS4 = qF211: variable at $K$ (Glass et al. 1999, 2001), indicative of WCLd+OB colliding wind binary. X-ray detection (Wang et al. (2006). Rotating IR pinwheel discovered (Tuthill et al. 2006), proving WC7-8d+OB colliding wind binary with $P=850 \pm 110 \mathrm{~d}$.

\section{Notes to Table 1}

Revised WR numbers of stars in 7Cat:

${ }^{1}$ : WR 77c: formerly WR 77b in NC03.

2 : WR 77e: formerly WR 77a in NC03.

3 : WR 77g: formerly WR 77c in NC03.

${ }^{4}$ : WR 77j: formerly WR 77e in NC03.

5 : WR 771: formerly WR 77d in NC03.

${ }^{6}$ : WR 77m: formerly WR 77f in NC03.

7 : WR 77n: formerly WR 77g in NC03. 
Table 1. continued.

\begin{tabular}{|c|c|c|c|c|c|c|c|c|c|c|c|}
\hline WR & $\begin{array}{l}\text { WR } \\
\text { discovery } \\
\text { designation }\end{array}$ & Other designation(s) & $\begin{array}{l}\quad \text { Discovery } \\
\text { in spectral } \\
\text { PG06 type }\end{array}$ & $\begin{array}{l}\text { Revised } \\
\text { spectral } \\
\text { type }\end{array}$ & ref. & $\begin{array}{l}m \\
(\mathrm{mag})\end{array}$ & ref. & RA(J2000) & $\operatorname{Dec}(\mathbf{J} 2000)$ & ref. & $\begin{array}{l}\text { WR } \\
\text { discovery } \\
\text { ref. }\end{array}$ \\
\hline
\end{tabular}

Galactic Center cluster

\begin{tabular}{|c|c|c|c|c|c|c|c|c|c|c|c|}
\hline $100 \mathrm{a}$ & & GC AF NW NW & E81 & WN7 & & & $K=12.6$ & PG06 & $174539.306-290030.68$ & $a$ & PG06 \\
\hline $101 a$ & $B S D 1$ & $M P E-8.3-5.7$ & E82 & $W C 9$ & WC8-9 & PG06 & $K=13.0$ & PG06 & $174539.382-290033.43$ & $a$ & $B S 95$ \\
\hline $101 b$ & $K G E 1$ & $A F N W$ & E74 & WN9-11 & WN8 & PG06 & $K=11.7$ & PG06 & $174539.458-290031.67$ & $a$ & $K G 95$ \\
\hline $101 c$ & $K G E 2$ & $A F$ & E79 & WN9-11 & Ofpe/WN9 & PG06 & $K=10.8$ & PG06 & $174539.541-290035.01$ & $a$ & $K G 95$ \\
\hline $101 d$ & $K G E 3$ & GC IRS 6E & & WC9 & & & $K=9.55$ & OE99 & $174539.643-290027.33$ & $b$ & $K G 95$ \\
\hline $101 \mathrm{da}$ & PGM 1 & & E60 & WN7? & & & $K=12.4$ & PG06 & $174539.708-290029.75$ & $a$ & PG06 \\
\hline $101 \mathrm{db}$ & PGM 2 & GC IRS 34W & E56 & Ofpe/WN9 & & & $K=11.4$ & PG06 & $174539.731-290026.51$ & $a$ & PG06 \\
\hline $101 \mathrm{dc}$ & MES-WR 1 & GC IRS 7SW & E66 & WN8-9 & WN8 & PG06 & $K=12.0$ & PG06 & $174539.739-290023.17$ & $a$ & ME05 \\
\hline $101 \mathrm{dd}$ & PGM 3 & GC IRS 34NW & E61 & WN7 & & & $K=12.8$ & PG06 & $174539.756-290025.25$ & $a$ & PG06 \\
\hline 101de & MPS 1 & GC IRS 13E5 & & WCLd? & & & $K=11.90$ & MP04 & $174539.780-290029.65$ & $c$ & MP04 \\
\hline $101 \mathrm{df}$ & MPS 2 & GC IRS 13E3B & & WCLd? & & & $K=13.07$ & MP04 & $174539.792-290029.59$ & $c$ & MP04 \\
\hline $101 \mathrm{dg}$ & TGM05-1 & GC IRS 2 , BSD96-45 & & WCLd? & & & $K=10.34$ & BS96 & $174539.792-290034.99$ & $i$ & TG05 \\
\hline $101 \mathrm{dh}$ & MPS 3 & GC IRS 13E3A & E49 & WCLd? & $?$ & PG06 & $K=13.0$ & PG06 & $174539.796-290029.63$ & $c$ & MP04 \\
\hline 101di & MPS 4 & GC IRS 13E4 & E48 & WC8-9 & WC9 & PG06 & $K=11.7$ & PG06 & $174539.797-290029.52$ & $a$ & MP04 \\
\hline $101 e^{12}$ & $K G E 5$ & GC IRS 13E2, MPS 5 & E51 & $W N 9-10+?$ & WN8 & PG06 & $K=10.8$ & PG06 & $174539.801-290029.84$ & $a$ & $K G 95$ \\
\hline 101ea & EML 1 & GC IRS 13E3c & & WCLd? & & & $K=12.49$ & MP04 & $174539.808-290029.48$ & EM04 & EM04 \\
\hline $101 f^{13}$ & $K G E 4$ & GC IRS 7W, MES-WR2 & E68 & WN9-10 & WC9 & PG06 & $K=13.1$ & PG06 & $174539.853-290022.11$ & $a$ & KG95 \\
\hline $101 \mathrm{fa}$ & HET 1 & GC IRS 3E & E58 & WC5-6d & WC5-6d? & PE05 & $K=15.0$ & PG06 & $174539.868-290024.30$ & $a$ & HE04 \\
\hline $101 \mathrm{~g}$ & $K G E 6$ & GC IRS 29N & E31 & WC9 & & & $K=10.0$ & PG06 & $174539.918-290026.69$ & $a$ & $K G 95$ \\
\hline $101 h^{14}$ & $K G E 8$ & GC IRS 15SW, MES-WR3 & E83 & WN9-11 & WN8-WC9 & PG06 & $K=12.0$ & PG06 & $174539.920-290018.08$ & $a$ & $K G 95$ \\
\hline $101 i^{15}$ & $K G E 7$ & GC IRS 29NE1, MPE-1.0-3.5 & E35 & WC9 & WC8-9 & PG06 & $K=11.7$ & PG06 & $174539.965-290026.04$ & $a$ & $K G 95$ \\
\hline $101 j$ & $K G E 9$ & GC IRS $16 N W$ & E19 & WN9-11 & Ofpe/WN9 & PG06 & $K=10.0$ & PG06 & $174540.042-290026.89$ & $a$ & $K G 95$ \\
\hline $101 \mathrm{ja}$ & PGM 4 & GC IRS 33E & E41 & Ofpe/WN9 & & & $K=10.1$ & PG06 & $174540.090-290031.22$ & $a$ & PG06 \\
\hline $101 k$ & $K G E 10$ & GCIRS $16 S W$ & E23 & WN9-11+? & Ofpe/WN9 & PG06 & $K=9.61 \mathrm{v}$ & GP00 & $174540.120-290029.08$ & $a$ & $K G 95$ \\
\hline 1011 & $K G E 11$ & GC IRS 16C & E20 & WN9-11 & Ofpe/WN9 & PG06 & $K=9.7$ & PG06 & $174540.126-290027.62$ & $a$ & $K G 95$ \\
\hline $101 \mathrm{~m}$ & $K G E 12$ & GC IRS 15NE & E88 & WN9-11 & WN8-9 & PG06 & $K=11.8$ & PG06 & $174540.145-290016.42$ & $a$ & KG95 \\
\hline $101 \mathrm{ma}$ & PGM 5 & & E71 & WC8-9? & & & $K=14.1$ & PG06 & $174540.161-290021.61$ & $a$ & PG06 \\
\hline $101 n$ & $K G E 13$ & GC IRS 16SE1, MPE+1.6-6.8 & E32 & WC9 & WC8-9 & PG06 & $K=10.9$ & PG06 & $174540.181-290029.25$ & $a$ & $K G 95$ \\
\hline 101 na & TGM02-1 & GC IRS 21 , BSD96-81 & & WCLd? & & & $K=10.55$ & CR01 & $174540.221-290030.84$ & $i$ & TG02 \\
\hline $101 \mathrm{nb}$ & PGM 6 & GC IRS 7SE2 & & WC? & & & $K=13.7$ & PG06 & $174540.245-200024.23$ & $k$ & PG06 \\
\hline $101 \mathrm{nc}$ & PGM 7 & GC IRS 9W & E65 & WN8 & & & $K=12.1$ & PG06 & $174540.257-290033.72$ & $a$ & PG06 \\
\hline $101 \mathrm{nd}$ & PGM 8 & GC IRS 16NE & E39 & Ofpe/WN9 & & & $K=8.9$ & PG06 & $174540.259-290027.07$ & $a$ & PG06 \\
\hline 1010 & $K G E 14$ & GC IRS 16SE2, MPE+2.7-6.9 & E40 & WC9 & WN5-6 & HE04 & $K=12.0$ & PG06 & $174540.264-290029.29$ & $a$ & $K G 95$ \\
\hline
\end{tabular}

8 : WR 77o: formerly WR 77h in NC03.

9 : WR 77p: formerly WR 77i in NC03.

${ }^{10}$ : WR 77r: formerly WR 77 in NC03.

11 : WR 77sc: formerly WR 77k in NC03.

12: WR 101e: formerly WR $101 f$ in 7 Cat.

Erratum: for GC IRS 13E1 in 7Cat, read GC IRS 13E2.

${ }^{13}$ : WR 101f: formerly WR 101e in 7Cat.

14: WR 101h: formerly WR 101i in 7Cat.

${ }^{15}$ : WR 101i: formerly WR $101 \mathrm{~h}$ in 7Cat.

16: WR 102bd: formerly WR 101q in HB03.

17: WR 102j: formerly WR $102 \mathrm{k}$ in 7Cat.

18: WR 102k: formerly WR $101 \mathrm{j}$ in 7Cat.

\section{Magnitudes:}

For each object the most recently published magnitude has been quoted, unless the new observation only confirms the earlier observation. CS99 used $K^{\prime}\left(\lambda_{\mathrm{c}}=2.11 \mu \mathrm{m}\right)$. FN02 used $m_{\mathrm{F} 205 \mathrm{w}}$. HB03 used $K_{\mathrm{s}}$ (narrow continuum filter $\lambda_{\mathrm{c}}=2.248 \mu \mathrm{m}$ ) from 2MASS.

\section{Coordinates:}

Coordinates from reference in last column, unless indicated otherwise (p.c. $=$ private communication):

$a$ : coordinates from F. Martins, 11 August 2005, p.c.; also PG05.

$b$ : revised coordinates from T. Paumard, October 2004, p.c. $c$ : coordinates from T. Paumard, August 2004, p.c.

$d$ : coordinates from CDS-Simbad. $e$ : coordinates from J. Moultaka, August 2005, p.c.

$f$ : coordinates from A. S. Cotera, July 2005, p.c. $g$ : coordinates from R. D. Blum, August 2004, p.c. $h$ : coordinates from D. F. Figer, August 2004, p.c.

$i$ : coordinates from F. Martins, 30 August 2005, p.c.; also PG05.

$j$ : coordinates from D. F. Figer, April 2006, p.c.

$k$ : coordinates from F. Martins, May 2006, p.c.

Reference abbreviations:

AR: Lang et al. (2001); Lang (2003), Lang et al. (2005).

BC94 = BCC: Bartaya et al. (1994).

BS01 = B = BSP: Blum et al. (2001).

BS95 = BSD: Blum et al. (1995).

BS96 = BSD96: Blum et al. (1996).

CE96 = C = CEC: Cotera et al. (1996).

CH06: Crowther et al. (2006).

CN02: Clark \& Negueruela (2002).

CP05 = CPG: Cohen et al. (2005).

CR01: Clénet et al. (2001).

CS99: Cotera et al. (1999).

DB04 = DBU: Drew et al. (2004).

E: running number in Paumard et al. (2006), Table 2.

EG01: Eikenberry et al. (2001).

EM04: Eckart et al. (2004).

EML = EML04: Eikenberry et al. (2004).

F: Figer et al. (2002). 
Table 1. continued.

\begin{tabular}{|c|c|c|c|c|c|c|c|c|c|c|c|c|}
\hline WR & $\begin{array}{l}\text { WR } \\
\text { discovery } \\
\text { designation }\end{array}$ & Other designation(s) & $\begin{array}{r}\text { in } \\
\text { PG06 }\end{array}$ & $\begin{array}{l}\text { Discovery } \\
\text { spectral } \\
\text { type }\end{array}$ & $\begin{array}{l}\text { Revised } \\
\text { spectral } \\
\text { type }\end{array}$ & ref. & $\begin{array}{l}m \\
\text { (mag) }\end{array}$ & ref. & RA(J2000) & $\operatorname{Dec}(\mathrm{J} 2000)$ & ref. & $\begin{array}{l}\text { WR } \\
\text { discovery } \\
\text { ref. }\end{array}$ \\
\hline
\end{tabular}

\section{Galactic Center cluster (continued)}

\begin{tabular}{|c|c|c|c|c|c|c|c|c|c|c|c|c|}
\hline 101oa & PMM 1 & HeI N3 & E59 & WR & WC9 & PG06 & $K=13.0$ & PG06 & 174540.264 & -290024.64 & $a$ & PM01 \\
\hline $101 \mathrm{ob}$ & PGM 9 & GC IRS 9SW & E76 & WC9 & & & $K=13.1$ & PG06 & 174540.366 & -290036.13 & $a$ & PG06 \\
\hline 101 oc & PMM 2 & GC IRS 7E2 (ESE) & E70 & WR & Ofpe/WN9 & PG06 & $K=12.9$ & PG06 & 174540.369 & -290022.76 & $b$ & PM01 \\
\hline 101od & TGM05-3 & GC IRS 5 & & WCLd? & & & & & 174540.4 & -290016 & $d$ & TG05 \\
\hline 101oe & MEV 1 & GC IRS 1W, BSD96-92 & & WCLd? & & & $K=8.72$ & CR01 & 174540.442 & -290027.53 & $i$ & ME04 \\
\hline 101 of & PGM 10 & GC IRS 9SE & E80 & WC9 & & & $K=11.7$ & PG06 & 174540.471 & -290036.27 & $a$ & PG06 \\
\hline $1010 g$ & TGM05-4 & GC IRS 10W, BSD96-94 & & WCLd? & & & $K=10.25$ & BS96 & 174540.49 & -290022.8 & OE99 & TG05 \\
\hline $101 \mathrm{oh}$ & PGM 11 & & E72 & WC9? & & & $K=13.6$ & PG06 & 174540.551 & -290028.60 & $a$ & PG06 \\
\hline $101 p$ & HBP 1 & & & WC8-9 & & & $K_{\mathrm{S}}=11.20$ & HB03 & 174542.47 & -285253.3 & HB03 & HB03 \\
\hline
\end{tabular}

\section{Arches cluster}

\begin{tabular}{|c|c|c|c|}
\hline $102 a$ & CSE 1 & "near G0.10+0.20" & $W N 8$ \\
\hline $102 \mathrm{aa}$ & NWS 1 & C13, AR6, B34, F2 & WN9 \\
\hline $102 \mathrm{ab}$ & BSP 30 & B30, F10 & WN7 \\
\hline $102 \mathrm{ac}$ & BSP 29 & B29, F17 & WN7 \\
\hline $102 \mathrm{ad}$ & NWS 4 & C9, AR3, B28, F1 & WN9 \\
\hline $102 \mathrm{ae}$ & NWS 5 & $\mathrm{C} 1, \quad \mathrm{~B} 26, \mathrm{~F} 9$ & WN9 \\
\hline 102af & NWS 6 & C3, AR16, B25, F12 & WN9 \\
\hline $102 \mathrm{ag}$ & NWS 7 & C6, AR2, B24, F8 & WN9 \\
\hline $102 \mathrm{ah}$ & NWS 8 & $\mathrm{C} 8, \mathrm{AR} 1, \mathrm{~B} 23, \mathrm{~F} 6$ & WN9 \\
\hline 102ai & NWS 9 & AR8, B22, F5 & WN9 \\
\hline 102aj & NWS 10 & $\mathrm{C} 5, \mathrm{AR} 4, \mathrm{~B} 21, \mathrm{~F} 7$ & WN9 \\
\hline 102ak & BSP 19 & B19, F16 & WN6-7 \\
\hline 102al & NWS 11 & $\mathrm{C} 2, \mathrm{AR} 5, \mathrm{~B} 17, \mathrm{~F} 4$ & WN9 \\
\hline $102 b$ & CSE 2 & "near SgrA East region A" & WN6 \\
\hline $102 \mathrm{ba}$ & CEC 7 & B12, F14 & WN7 \\
\hline $102 \mathrm{bb}$ & NWS 14 & $\mathrm{C} 11, \mathrm{AR} 7, \mathrm{~B} 3, \mathrm{~F} 3$ & WN9 \\
\hline $102 b c$ & CEC 10 & B1 & WN7 \\
\hline
\end{tabular}

\begin{tabular}{|c|c|c|c|c|c|c|c|}
\hline & & $K^{\prime}=10.22$ & CS99 & 174548.560 & -285006.08 & $f$ & CS99 \\
\hline \multirow[t]{3}{*}{ WN9+OB? } & LG01 & $K^{\prime}=10.7$ & CE96 & 174549.76 & -284926.0 & LJ05 & NW95 \\
\hline & & $K^{\prime}=11.46$ & FNO2 & 174550.08 & -284926.2 & $g$ & BS01 \\
\hline & & $K^{\prime}=12.15$ & FN02 & 174550.15 & -284926.9 & $g$ & BS01 \\
\hline \multirow[t]{4}{*}{ WN9+OB? } & LJ05 & $K^{\prime}=10.2$ & CE96 & 174550.20 & -284922.3 & LJ05 & NW95 \\
\hline & & $K^{\prime}=10.6$ & CE96 & 174550.31 & -284911.5 & $g$ & NW95 \\
\hline & & $K^{\prime}=10.6$ & CE96 & 174550.31 & -284917.0 & $g$ & NW95 \\
\hline & & $K^{\prime}=10.76$ & FN02 & 174550.39 & -284921.3 & LJ05 & NW95 \\
\hline WN9+OB? & LJ05 & $K^{\prime}=10.1$ & CE96 & 174550.42 & -284922.3 & LJ05 & NW95 \\
\hline WN9+OB? & LJ05 & $K^{\prime}=10.86$ & FN02 & 174550.45 & -284931.9 & LJ05 & NW95 \\
\hline \multirow[t]{2}{*}{ WN9+OB? } & LJ05 & $K^{\prime}=9.7$ & CE96 & 174550.47 & -284919.5 & LJ05 & NW95 \\
\hline & & $K^{\prime}=11.40$ & FN02 & 174 & -28 & $g$ & BS01 \\
\hline \multirow[t]{3}{*}{ WN8 } & $\mathrm{La03}$ & $K^{\prime}=10.2$ & CE96 & 174550.57 & -284917.5 & LJ05 & NW95 \\
\hline & & $K^{\prime}=10.97$ & CS99 & 174550.626 & -285919.61 & $f$ & CS99 \\
\hline & & $K^{\prime}=11.22$ & FN02 & 174550.69 & -284922.5 & $g$ & CE96 \\
\hline \multirow[t]{2}{*}{ WN9/Ofpe } & $\mathrm{La03}$ & $K^{\prime}=10.3$ & CE96 & 174550.83 & -284926.4 & LJ05 & NW95 \\
\hline & & $K^{\prime}=11.3$ & CE96 & 174551.46 & -284926.0 & $g$ & CE96 \\
\hline
\end{tabular}

FM95: Figer et al. (1995).

FM96: Figer et al. (1996).

FM99a $=$ FMM = FMM99: Figer et al. (1999a).

FM99b = FMG99: Figer et al. (1999b).

FN05 = FNG: Figer et al. (2005).

GCS: Nagata et al. (1995).

GD06 = GDTB: Groh et al. (2006).

GM99: Glass et al. (1999).

GP00 = GPE: Genzel et al. (2000).

HB03 = HBP: Homeier et al. (2003).

HB05 = HBD: Hopewell et al. (2005).

HE04 = HET: Horrobin et al. (2004).

KG95 = KGE: Krabbe et al. (1995).

La03: Lang (2003).

LG01: Lang et al. (2001).

LJ05: Lang et al. (2005).

ME04 = MEV: Moultaka et al. (2004).

ME05 = MES: Moultaka et al. (2005).

MP04 = MPS: Maillard et al. (2004).

NC03: Negueruela \& Clark (2003).

NC05 = NC: Negueruela \& Clark (2005).

Ne03: Negueruela (2003).

Ne05: Negueruela, priv. comm.: VLT-FORS spectroscopy.

NW95 = NWS: Nagata et al. (1995).

OE99: Ott et al. (1999).

Pa04: Paumard (2004, private communication.

PC02 = PCG: Pasquali et al. (2002).
PE05: Pott et al. (2005).

PG04: Paumard et al. (2004).

PG05: Paumard et al. (2005).

PG06 = PGM: Paumard et al. (2006).

PM01 = PMM: Paumard et al. (2001).

PM03: Paumard et al. (2003).

$\mathrm{Q}=\mathrm{GMM}=$ GM90: Glass et al. (1990) (see also Moneti et al. 2001).

QR: Lang et al. (1999); Lang (2003); Lang et al. (2005).

TG02 = TGM02: Tanner et al. (2002).

TG05 = TGM05: Tanner et al. (2005).

TM06: Tuthill et al. (2006).

\section{Conclusion}

The past five years have seen the number of known Galactic WR stars increasing by $\sim 30 \%$ to close to 300 objects. It is to be expected that, with the advance of observing capabilities, that number will continue to increase. Whether the expected number of $\sim 1600$ WR stars in our observable quadrant of the Galaxy (van der Hucht 2001) will be reached remains to be seen.

Discovering and monitoring WR star in the Galaxy and in the Local Group is important for the study of Galactic structure and chemical evolution, and it is likely that some WR 
Table 1. continued.

\begin{tabular}{|c|c|c|c|c|c|c|c|c|c|c|c|}
\hline WR & $\begin{array}{l}\text { WR } \\
\text { discovery } \\
\text { designation }\end{array}$ & $\begin{array}{l}\text { Other } \\
\text { designation(s) }\end{array}$ & $\begin{array}{l}\text { Discovery } \\
\text { spectral } \\
\text { type }\end{array}$ & $\begin{array}{l}\text { Revised } \\
\text { spectral } \\
\text { type }\end{array}$ & ref. & $\begin{array}{l}m \\
(\mathrm{mag})\end{array}$ & ref. & $\mathrm{RA}(\mathrm{J} 2000)$ & $\operatorname{Dec}(\mathbf{J} 2000)$ & ref. & $\begin{array}{l}\text { WR } \\
\text { discovery } \\
\text { ref. }\end{array}$ \\
\hline $102 b d^{16}$ & HBP 2 & & WC8-9 & & & $K_{\mathrm{s}}=11.49$ & HB03 & 174557.78 & -285446.1 & $\mathrm{HB} 03$ & HB03 \\
\hline
\end{tabular}

\section{Quintuplet cluster}

\begin{tabular}{|c|c|c|c|c|c|c|c|c|c|c|}
\hline $102 c$ & FMM96-1 & $q F 353 E$ & WN6 & & $K=11.53$ & FM99 & 174611.2 & -284905.6 & 7Cat & FM95 \\
\hline $102 \mathrm{ca}$ & HBP 3 & & WC8-9 & & $K_{\mathrm{s}}=10.40$ & HB03 & 174613.04 & -284925.4 & HB03 & HB03 \\
\hline $102 d$ & FMM95-1 & $q F 320$ & WN9 & & $K=10.50$ & FM99 & 174614.067 & -284917.28 & $h$ & FM95 \\
\hline $102 \mathrm{da}$ & FMM-d1 & GCS 3-4, qF243, Q1 & WCLd? & & $K=7.61$ & GM99 & 174614.151 & -284937.42 & $h$ & FM96 \\
\hline $102 \mathrm{db}$ & FMM-d2 & GCS 3-3, qF258, Q9 & WCLd? & & $K=8.98$ & GM99 & 174614.336 & -284932.17 & $h$ & FM96 \\
\hline $102 \mathrm{dc}$ & FMM-d3 & GCS 3-2, qF231, Q2, QR7 & WCLd? & WC7-8d+OB TM06 & $K=6.28 \mathrm{v}$ & GM99 & 174614.721 & -284941.46 & $h$ & FM96 \\
\hline $102 d d$ & FMM-d4 & GCS 3-1, qF251, Q4 & WCLd? & & $K=7.66$ & GM99 & 174614.810 & -284935.02 & $h$ & FM96 \\
\hline $102 e$ & FMM96-2 & $q F 151$ & $W C 8$ & & $K=10.44$ & FM99 & 174614.827 & -285001.17 & $h$ & FM96 \\
\hline $102 \mathrm{ea}$ & FMM96-7 & $\mathrm{qF} 241, \mathrm{Q} 10, \mathrm{QR} 5$ & WN9/Ofpe & & $K=8.83$ & GM99 & 174615.129 & -284937.82 & $j$ & FM96 \\
\hline $102 f$ & FMM96-3 & $q F 235 N$ & $W C<8+?$ & & & & 174615.168 & -284940.25 & $h$ & FM96 \\
\hline $102 \mathrm{~g}$ & FMM99-1 & $q F 235 S$ & $W C<8$ & & & & 174615.182 & -284942.40 & $h$ & FM99 \\
\hline $102 \mathrm{~h}$ & FMM95-2 & $q F 76$ & WC9 & & $K=11.44$ & FM99 & 174615.572 & -285018.89 & $h$ & FM95 \\
\hline 102ha & FMM-d5 & GCS 4, qF211, Q3 & WCLd? & $\mathrm{WCLd}+\mathrm{OB}$ & $K=6.91 \mathrm{v}$ & GM99 & 174615.884 & -284946.27 & $h$ & FM96 \\
\hline $102 \mathrm{hb}$ & FMM96-8 & $\mathrm{qF} 240, \mathrm{Q} 8$ & WN9/Ofpe & & $K=9.01$ & GM99 & 174615.954 & -284938.60 & $j$ & FM96 \\
\hline $102 i$ & FMM96-4 & $q F 256$ & $W N 9+?$ & & $K=11.38$ & FM99 & 174616.560 & -284932.53 & $h$ & FM96 \\
\hline $102 j^{17}$ & FMM96-6 & $q F 309$ & $W C<8$ & & $K=11.52$ & FM99 & 174617.522 & -284919.41 & $h$ & FM96 \\
\hline $102 k^{18}$ & FMM96-5 & $q F 274$ & WN9 & & $K=11.41$ & FM99 & 174617.548 & -284929.52 & $h$ & FM96 \\
\hline $102 \mathrm{ka}$ & HBP 4 & & WN10 & & $K_{\mathrm{s}}=8.84$ & HB03 & 174618.12 & -290136.5 & $\mathrm{HB} 03$ & HB03 \\
\hline
\end{tabular}

\section{"SGR 1806-20" cluster}

\begin{tabular}{|c|c|c|c|c|c|c|c|c|}
\hline $111 \mathrm{a}$ & FNG 1 & FNG-1 & WC8 & $K=11.76$ & FN05 & 180838.32 & -202433.5 & FN05 FN05 \\
\hline $111 b$ & EGH 1 & FNG-B & WC9d & $K=10.50$ & FN05 & 180839.24 & -202442.50 & FN05 EG01, EML04 \\
\hline $111 \mathrm{c}$ & FNG 2 & FNG-2 & WN6 & $K=12.16$ & FN05 & 180839.42 & -202442.57 & FN05 FN05 \\
\hline $111 d$ & FNG 3 & FNG-3 & WN7? & $K=12.87$ & FN05 & 180839.50 & -202435.88 & FN05 FN05 \\
\hline $142 \mathrm{a}$ & PCG 1 & NGC 6910-MS 21 & WC8 & $K_{\mathrm{s}}=7.09$ & PC02 & 202406.2 & +412533 & PC02 PC02 \\
\hline 159 & BCC 1 & $\mathrm{BD}+62^{\circ} 2296 \mathrm{~B}$ & WN4 & $V_{T}=11.20$ & $\mathrm{Ne} 03$ & 234720.4 & +631314 & $\mathrm{Ne} 03$ BC94, Ne03 \\
\hline
\end{tabular}

stars are Type Ib/c supernova progenitors and/or GRB progenitors. Identifying even one such object before it explodes could contribute greatly to our understanding of these energetic phenomena.

Acknowledgements. The author is much indebted to Drs. Bob Blum, Angela Cotera, Paul Crowther, Don Figer, Ella Hopewell, Jessica LaVine, Fabrice Martins, Jihane Moultaka, and Thibaut Paumard for providing data on new WR stars in advance of publication, for re-determining coordinates of 7Cat WR stars in crowded regions, and for helpful comments and suggestions. Constructive comments and suggestions from the referee are highly appreciated.

\section{References}

Baganoff, F. K., Maeda, Y., Morris, M., et al. 2003, ApJ 591, 891 Bartaya, R. A., Chargeishvili, K. B., Chentsov, E. L., \& Shkhagosheva, Z. U. 1994, Bull. Special Astrophys. Obs. 38, 103

Blum, R. D., Sellgren, K., \& DePoy, D. L. 1995, ApJ, 440, L17

Blum, R. D., Sellgren, K., \& DePoy, D. L. 1996, ApJ, 470, 864

Blum, R. D., Schaerer, D., Pasquali, A., et al. 2001, AJ, 122, 1875

Chevalier, R. A. 2005, ApJ, 619, 839

Clark, J. S., \& Negueruela, I. 2002, A\&A, 396, L25

Clark, J. S., Negueruela, I., Crowther, P. A., \& Goodwin, S. P. 2005, A\&A, 434, 949

Clénet, Y., Rouan, D., Gendron, E., et al. 2001, A\&A, 376, 124

Cohen, M., Parker, Q. A., \& Green, A. J. 2005, A\&A, 360, 1439
Cotera, A. S., Erickson, E. F., Colgan, S. W. J., et al. 1996, ApJ, 461, 750

Cotera, A. S., Simpson, J. P., Erickson, E. F., et al. 1999, ApJ, 510, 747

Crowther, P. A., Hadfield, L. J., Clark, J. S. Negueruela, I., \& Vacca, W. D. 2006, MNRAS, in press [arXiv:astro-ph/0608356]

DePoy, D. L., Pepper, J., Pogge, R. W., Stutz, A., Pinsonneault, M., \& Sellgren, K. 2004, ApJ, 617, 1127

Drew, J. E., Barlow, M. J., Unruh, Y. C., et al. 2004, MNRAS, 351, 206

Dwarkadas, V. 2005, ApJ, 630, 892

Eckart, J., Moultaka, J., Viehmann, T., Straubmeier, C., \& Mouawad, N. 2004, ApJ, 602, 760

Eikenberry, S. S., Garske, M. A., Hu, D., et al. 2001, ApJ, 563, L133

Eikenbery, S. S., Matthews, K., LaVine, J. L., et al. 2004, ApJ, 616, 506

Eldridge, J. J., \& Vink, J. S. 2006, A\&A, 452, 295

Eldridge, J. J., Genet, F., Daigne, F., \& Mochkovitch, R. 2006, MNRAS, 367, 186

Figer, D. F., McLean, I. S., \& Morris, M. 1995, ApJ, 447, L29

Figer, D.F., Morris, M., \& McLean, I.S. 1996, in The Galactic Center, ed. R. Gredel, Proc. 4th ESO/CTIO Workshop, La Serena, Chile, 10-15 March 1996, ASP-CS 102, 263

Figer, D. F., McLean, I. S., \& Morris, M. 1999a, ApJ, 514, 202

Figer, D. F., Morris, M., Geballe, T. R., et al. 1999b, ApJ, 525, 759

Figer, D. F., Najarro, F., Gilmore, D., et al. 2002, ApJ, 581, 258

Figer, D. F., Najarro, F., Geballe, T. R., Blum, R. D., \& Kudritzki, R. P. 2005, ApJ, 622, L49

Fruchter, A. S., Levan, A. J., Strolger, L., et al. 2006, Nature, 441, 463

Geballe, T. R., Najarro, F., Rigaut, F., \& Roy, J.-R. 2006, ApJ, in press, 10 November 2006 [arXiv:astro-ph/0607550]

Genzel, R., Pichon, C., Eckart, A., Gerhard, O. E., \& Ott, T. 2000, MNRAS, 317, 348 
Glass, I. S., Moneti, A., \& Moorwood, A. F. M. 1990, MNRAS, 242, 55P (Erratum: MNRAS 244, 767)

Glass, I. S., Matsumoto, S., Carter, B. S., \& Sekiguchi, K. 1999, MNRAS, 304, L10

Glass, I. S., Matsumoto, S., Carter, B. S., \& Sekiguchi, K. 2001, MNRAS, 321, 77

Groh, J. H., Damineli, A., Teodoro, M., \& Barbosa, C. L. 2006, A\&A, 457, 591

Hammer, F., Flores, H., Schaerer, D., et al. 2006, A\&A, 454, 103

Hirschi, R., Meynet, G., \& Maeder, A. 2005, A\&A, 443, 581

Homeier, N. L., Blum, R. D., Pasquali, A., Conti, P. S., \& Damineli, A. 2003, A\&A, 408, 153

Hopewell, E. C., Barlow, M. J., Drew, J. E., et al. 2005, MNRAS, 363, 857

Horrobin, M., Eisenhauer, F., Tecza, M., et al. 2004, AN, 325, 88

van der Hucht, K. A. 2001, New Astron. Rev., 45, 135 (7Cat)

Krabbe, A., Genzel, R., Eckart, A., et al. 1995, ApJ, 447, L95

Lang, C. C. 2003, in A Massive Star Odyssey, from Main Sequence to Supernova, ed. K. A. van der Hucht, A. Herrero \& C. Esteban, Lanzarote, Canary Islands, 24-28 June 2002 (San Francisco: ASP), Proc. IAU Symp., 212,497

Lang, C. C., Figer, D. F., Goss, W. M., \& Morris, M. 1999, AJ, 118, 2327

Lang, C. C., Goss, W. M., \& Rodríguez, L. F. 2001, ApJ, 551, L143

Lang, C. C., Johnson, K. E., Goss, W. M., \& Rodríguez, L. F. 2005, AJ, 130, 2185

Langer, N., \& Norman, C. A. 2006, ApJ, 638, L63

Law, C., \& Yusef-Zadeh, F. 2004, ApJ, 611, 858

Maillard, J. P., Paumard, T., Stolovy, S. R., \& Rigaut, F. 2004, A\&A, 423, 155

Marston, A. P. 2005, ApJ, 475, 188

Meynet, G., \& Maeder, A. 2005, A\&A, 429, 581

Moneti, A., Stolovy, S., Blommaert, J. A. D. L., Figer, D. F., \& Najarro, F. 2001, A\&A, 366, 106

Moultaka, J., Eckart, A., Viehmann, T., et al. 2004, A\&A, 425, 529

Moultaka, J., Eckart, A., Schödel, R., Viehmann, T., \& Najarro, F. 2005, A\&A, 443,163

Muno, M. P., Bower, G. C., Burgasser, A. J., et al. 2006, ApJ, 638, 183

Nagata, T., Woodward, C. E., Shure, M., \& Kobayashi, N. 1995, AJ, 109, 1676
Negueruela, I. 2003, A\&A, 408, 689

Negueruela, I. 2005, private communication

Negueruela, I., \& Clark, J. S. 2003, in A Massive Star Odyssey, from Main Sequence to Supernova, ed. K. A. van der Hucht, A. Herrero, \& C. Esteban, Lanzarote, Canary Islands, 24-28 June 2002 (San Francisco: ASP), Proc. IAU Symp., 212, 531

Negueruela, I., \& Clark, J. S. 2005, A\&A, 436, 541

Ott, Th., Eckart, A., \& Genzel, R. 1999, ApJ, 523, 248

Parker, Q. A., Phillipps, S., Pierce, M. J., et al. 2005, MNRAS, 362, 689

Pasquali, A., Comerón, F., Gredel, R., Torra, J., \& Figuerras, F. 2002, A\&A, 396, 533

Paumard, T., Maillard, J. P., Morris, M., \& Rigaut, F. 2001, A\&A, 366, 466

Paumard, T., Maillard, J.-P., \& Stolovy, S. 2003, Astron. Nachr., 324, 303

Paumard, T., Maillard, J.-P., \& Morris, M. 2004, A\&A, 426, 81

Paumard, T., Genzel, R., Maillard, J. P., et al. 2005, in Young Local Universe, ed. A. Chalabaev, F. Fukui, T. Montmerle, \& J. Tran-Than-Van, Proc. XXXIXth Rencontres de Moriond, La Thuile, Aosta Valley, Italia, 21-28 March 2004 (Paris: Édition Frontières), 377

Paumard, T., Genzel, R., Martins, F., et al. 2006, ApJ, 643, 1011

Petrovic, J., Langer, N., Yoon, S.-C., \& Heger, A. 2005, A\&A, 435, 247

Pott, J. U., Eckart, A., Glindemann, A., et al. 2005, The ESO Messenger, 119, 43

Skinner, S. L., Simmons, A. E., Zhekov, S. A., et al. 2006, ApJ, 639, L35

Tanner, A., Ghez, A. M., Morris, M., et al. 2002, ApJ, 575, 860

Tanner, A., Ghez, A. M., Morris, M. R., \& Christou, J. C. 2005, ApJ, 624, 742

Tanner, A., Figer, D. F., Najarro, F., et al. 2006, ApJ, 641, 891

Trippe, S., Martins, F., Ott, T., et al. 2005, A\&A, 448, 305

Tuthill, P. G., Monnier, J. D., Tanner, A., et al. 2006, Science, 313, 935

Viehmann, T., Eckart, A., Schödel, R., Pott, J.-U., \& Moultaka, J. 2006, ApJ, 642,861

Wang, Q. D., Dong, H., \& Lang, C. 2006, MNRAS, 371, 38

Williams, P. M., van der Hucht, K. A., \& Thé, P. S. 1987, A\&A, 182, 91

Woosley, S. E., \& Heger, A. 2006, ApJ, 637, 914

Yoon, S.-C., \& Langer, N. 2005, A\&A, 443, 643

Zou, Y. C., Wu, X. F., \& Dai, Z. G. 2005, MNRAS, 363, 93 